\title{
Improving Vehicular Mobility in Urban Traffic Using Ubiquitous Computing
}

\author{
J. A. Castán1, S. Ibarra Martínez¹, J. Laria Menchaca1, M. G. Treviño Berrones1, \\ J. Pérez Cobos ${ }^{1}$, E. Castán Rocha ${ }^{2}$ \\ ${ }^{1}$ Faculty of Engineering, Autonomous University of Tamaulipas, Victoria City, Mexico \\ ${ }^{2}$ Madero Institute of Technology, Tamaulipas, Mexico \\ Email: jacastan@uat.edu.mx
}

Received 25 June 2016; accepted 14 August 2016; published 17 August 2016

Copyright (C) 2016 by authors and Scientific Research Publishing Inc.

This work is licensed under the Creative Commons Attribution International License (CC BY).

http://creativecommons.org/licenses/by/4.0/

c) (i) Dpen Access

\section{Abstract}

The paper introduces a novel paradigm to use ubiquitous computing in urban traffic control as a methodology to include the benefits of considering physical elements implicated in the environment. This promising idea arises from previous results in the literature, where ubiquitous computing achieves significant and promising results in diverse scenarios. Some works in the state-ofthe-art clearly state that traditional traffic light signals are not capable of offering higher service levels when they should control the vehicular mobility because of different constraints. In this sense, the paper proposes an adaptation of the main ideas of ubiquitous computing as a metaphor to facilitate the interaction between users and traffic infrastructures in order to improve the users' experience on the road.

\section{Keywords}

Ubiquitous Computing, Urban Traffic Control, Intelligent Transport System

\section{Introduction}

About 25 years ago, Mark Weiser [1] introduces a new paradigm of an emergent advance computing technique, trying to offer proper solution to open and distribute problems in real scenarios. Such proposal was called Ubiquitous Computing. After 10 year of the Weiser's proposal, Mahadev Satyanarayanan introduces a novel perspective of the ubiquitous computing called Pervasive Computing [2]. Such tends were a motivation for a great number of researches along the world, because of their wide range of operatively; and their easier adaptability to solve requirements and constraints of dynamic and uncertain situations. In fact, ubiquitous computing refers to a

How to cite this paper: Castán, J.A., Ibarra Martínez, S., Laria Menchaca, J., Treviño Berrones, M.G., Pérez Cobos, J. and Castán Rocha, E. (2016) Improving Vehicular Mobility in Urban Traffic Using Ubiquitous Computing. Journal of Computer and Communications, 4, 57-62. http://dx.doi.org/10.4236/jcc.2016.410006 
world where devices (i.e., computers, mobile devices, etc.) aim to improve the quality of the life of users without being noticed. For instance, we can assume that each device of a house is tagged by the users, and based on a context; the devices can react in a proper way, to the conditions of the house to offer a suitable ambient to the habitants or visitants.

Ubiquitous computing has found application in productive services such as: health [3], commerce [4], competence management [5], learning [6], logistic [7] [8], urban traffic [9], telecommunication [10], games [11], etc.

To do this, in [12], the author suggested that the proliferation of the smartphones and tablets fostered an ample adoption of Mobile Computing. By using GPS technology, the aforementioned devices are useful to endow users with a robust way to communicate a specific set of information to other users or systems without being noticed.

The increasing use of such technology (i.e., sensors) has enabled the availability of computational service on a specific context in order to offer solutions that increase the users' quality of life.

To follow, Section 2 presents a general overview of implementing ubiquitous computing in intelligent transport and transportation systems. Besides, Section 3 is devoted to present the general scheme of the proposal of this paper. To the end, in Section 4, conclusions and future work are depicted in order to highlight the advantages of implementing ubiquitous computing to control, manage and optimize urban traffic in modern cities.

\section{Ubiquitous Computing in Intelligent Transport and Transportation Systems}

The great relevance of urban traffic depends on the rapid growth of the urban population. Unfortunately, Mexico is a country that has presented an exponential demographic development, which has not submitted regulatory arrangements to control the use and acquisition of vehicles to domestic users. In light of this, some technologies have been adopted, as a reactive response to the current requirements and constrains. For example, the traffic light signals are controlled by experts in a remote way using SCATS ${ }^{1}$ in one of the most relevant zone of Mexico City. Even though, it was a suitable measure, such response has not been fully successful. Some problems have not taken into account yet.

For example, SCATS is an urban traffic system that relies on the criterion of control experts, and we know that this fact must consider the possibility (in a very wide margin) of human error.

Therefore, intelligent transport systems are a useful paradigm that joint advanced computational techniques with some traditional urban traffic control models.

In light of this, the reference [13] presents an innovative evolutionary approach for optimizing public transportation planning, taking into account practical constraints (the duration trip between stops and capacities of vehicles used), has been developed and validated on several examples. Thanks to these constraints, the model fits very well to the real-world vehicle operations data. The future research work will consist on developing agent-based systems to control the traffic in the second step i.e. the transportation exploitation. In fact, during the functioning of systems, disturbances can reduce the quality of service, therefore, it will be essential to regulate the traffic by optimizing the transit time and the waiting time of passengers in stops in order to improve this quality of service.

Besides, in [14] the authors design and develop a UQTI (Ubiquitous Query for Travel Information) information system to enhance the service quality of transport system. To support the service platform, they develop a partially connected routing protocol. Moreover, they offer a systematic approach to evaluate the connectivity availability of such UQTI. By modeling and simulation, the authors find that as compared to the full connected routing protocol, the partially connected routing design can achieve much higher packet delivery ratio in network with poor connectivity with tolerant delay. A demonstration system of UQTI is developed to validate the feasibility of the system. This work helps to achieve network energy efficiency, reliability, and timeliness in an operable fashion.

Reference [15] has been identified the information lifecycle in city-wide ubiquitous computing environment for effective information management and separated this lifecycle into three phases: data acquisition, complex event processing and information delivery. In the lifecycle of data acquisition, the paper describes the characteristics of the data gathered in ubiquitous environment based on actual cases in South Korea and addressed the issue to be considered in this stage. In the lifecycle of complex event processing, the authors describe the event processing mechanism by which information or data delivered from the first lifecycle is selected as an event that is meaningful to the business domain by using CEP technology. The paper also introduced the metadata for event in XML format. In the lifecycle of information delivery, we suggest the message format for a patterned

${ }^{1}$ http://www.scats.com.au. 
protocol in sending and receiving message from the second lifecycle. But more work is needed. Finally, the conclusions argue that it is necessary to evaluate performance when adopting this information lifecycle management to many cities.

The reference [16] presents a novel theoretical three-tier architecture to locate on-street parking space while the driver is still driving the vehicle. The intelligent parking detection mechanism makes use of wireless sensor networks to gather context information describing the driver, vehicle and the environmental conditions. The gathered context information is transmitted to the wireless InfoStation, which intelligently processes the context information to locate a vacant parking space. Swarm Intelligence is proposed to calculate the parking space at an optimum distance from the location requested by the driver. Intelligent parking detection and reservation is an emerging area of research aided with computational intelligence paradigms. VANET based technology enables communication between vehicles and infrastructure to locate parking. The goal of intelligent parking systems is to let the driver know where parking is available, making it easier for cars to find their way into parking spots. Another goal is to understand where parking is more in demand and during which time intervals. The proposed architecture indicates the importance of context in parking location and reservation. The proposed architecture warrants further investigation into the applicability of swarm intelligence along with Network Simulator-3 (NS-3) and the contributions of this paper are expected to lead to advances in the design and control of WSN and SI based intelligent parking space location.

In [17] the authors explain their personal point of view about the Smart City idea, in the context of transportation of citizens. The paper presents the main principles and techniques used and presented a precise application based on IoT and LBS. Before in-the-field deployment of this kind of system, it is important to validate it theoretically from technological and human acceptability points of view. For that reason, the authors created a simulator allowing them to design and simulate different scenarios of infrastructure behaviors. They also use this simulator for user acceptability studies, the goal of which is to validate (or invalidate) acceptability of new driving situations from the driver's point of view. For experimental reasons, they tested the user's (driver's) behavior in relation with new vertical and horizontal road signs. They comment that the next step is a vertical(driver's top) view proposed by the simulator, allowing them to study the dynamic driver's reactions related to vertical and horizontal road signs depending on the behavior of other vehicles around the driver.

Along this section, the paper presents relevant and significant contributions of ubiquitous computing to intelligent transport and transportation systems, which represent a promising path to reach intelligent ambient in modern and progressive cities.

\section{Our Proposal}

In recent years, road traffic has become an essential part of a modern city. Long lines of vehicles, wide ranges of stop-times in intersections, environmental emissions, priority to service vehicle, and pedestrian needs, are only some of the aspects that traffic control is looking forward to eliminating. The transportation community has developed a great interest in bringing forth techniques to represent, model and assess traffic situations in interconnected transport networks [9].

Figure 1 depicted the general scheme of the Ubiquitous Urban Traffic Control UUTC methodology proposed in this paper, which consists of three fundamental components; 1) cloud-computing techniques which are devoted to process the information provided by the follow two components, in order to administrate the general behavior of the traffic light signals and to compute relevant information about the green house emissions; 2) mobile devices which manage and communicate the information of the users with the cloud; and 3) urban infrastructure which represent the physical change over the instruments at the infrastructure.

\subsection{Cloud-Computing Techniques}

To ensure a high level of service in urban roads, which assesses a better vehicular mobility and a considerable reduction of greenhouse gases emission, the paper proposes the use of some cloud-computing techniques. Such techniques arise as a proper solution to provide a suitable service to users on the way without the user intervention. In this sense, the cloud receives a set of information provided by diverse kind of devices and must react to decide what actions must the traffic light signals do. The possible process that cloud-computing must done are listened as follow:

- To define if it is necessary to modify the order of the phase.

- To set the time of green light interval. 


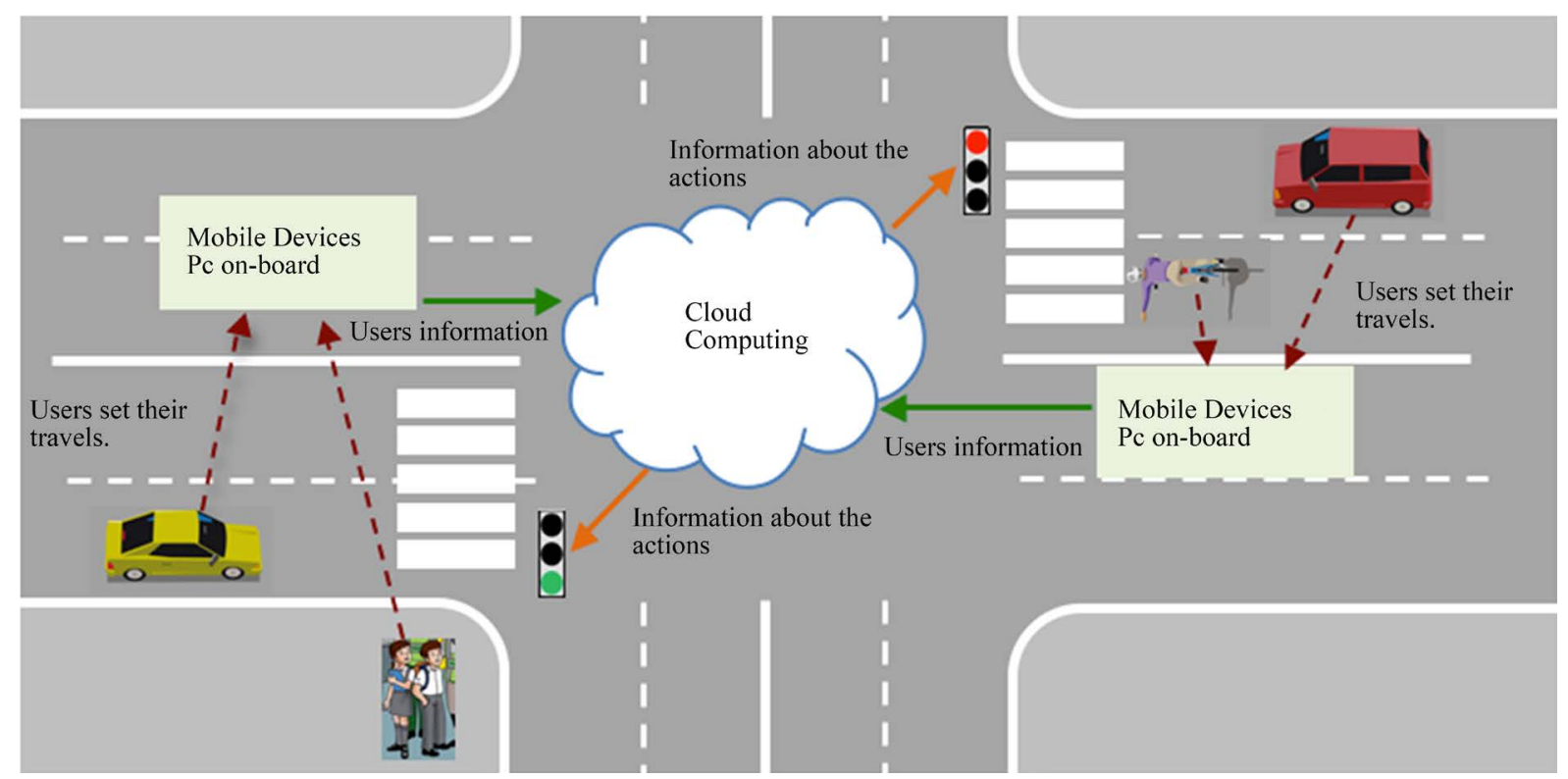

Figure 1. General scheme of the methodology.

- To compute the number of car passing through the intersection.

- To create a classification of the cars by size.

- To estimate the generation of the greenhouse gases.

Implementing a specific cloud computing technique can develop all these functionalities. For instance, neural networks or genetic algorithms are approaches able to consider real-time information into their decision-making structure, such as human being does. These systems are robust enough to withstand uncertainty without compromising the specificity of the decisions to be taken. On the other hand, fuzzy logic or neuro-fuzzy logic tends to be suitable options to sort cars depending on a well-defined set of attributes.

\subsubsection{Mobile Devices}

Mobile devices play one of the most important roles of this proposal because they are the responsible for moving the users information to the cloud. There are various technologies, which are capable of encoding and sending data to the cloud in a real-time, without the user having to worry about performing this process. Mobile phones and some vehicles are now equipped with technologies and applications that try to raise the functionalities that are offered to users.

For example, radio frequency identification RFID is a system that cans storage and recovery information in a remote way using tags.

Moreover, global positioning systems GPS is defined as a space-based navigation system that provides location and time information under any weather condition and wherever the user is, obviously when the user can be seen by the satellite.

Recent advances have demonstrated the effectiveness of these technologies in different application environments where information in fully dynamic [18] [19].

Specifically, the paper defines that the mobile devices send information about the user (i.e., vehicles, pedestrians, cyclists, etc) on the road to the cloud. All information that mobile devices will send is listened as follow:

- for vehicles:

o velocity,

o direction,

o type of car,

o internal services actived (i.e., air conditioner),

$\mathrm{o}$ if it is a service vehicle (i.e., ambulance, fire truck, police, etc.),

o if the car is in troubles (i.e., crashed, broken dawn, etc).

- for pedestrians: 
o position,

o route,

o physical capabilities.

- for cyclist:

o velocity,

o route,

o age.

\subsubsection{Urban Infrastructure}

An urban infrastructure refers to the set of devices and conditions that shape the intersection. Normally, traffic lights signals and traffic signals are two of the most relevant ways to inform to users about the permission over the road.

The main objective of this proposal is based on the idea that the cloud is able to control and modify in real time, the duration of a particular light on the traffic light signals according to the conditions and requirements on the road.

To do this, the traffic light signals must be updated; this means that a new generation of these devices must be created, so that they are able to receive instructions and modify its programming times.

For example, Arduino ${ }^{2}$ and RaspberryPi ${ }^{3}$ are electronic devices capable of offering embedded solutions for developing a sophisticated traffic light signal. These two options can interact with other computers or mobile devices by using WiFi or Bluetooth communication.

\section{Conclusions and Future Work}

From the delineation of the fundamental ideas of ubiquitous computing, several technologies have been used in the implementation of ubiquitous systems using diverse mobile devices as efficient tools to facilitate and improve the users' quality of life. Some trends aim to build applications that allow users to enjoy the banalities of lives, while devices and systems work for them.

The paper introduces an innovative evolutionary methodology for optimizing urban traffic called Ubiquitous Urban Traffic Control UUTC, which takes advantages of the benefits of ubiquitous computing in intelligent transport and transportation systems. Such paradigm permits the implementation of real-life rules similar to those people usually resort to.

Ubiquitous computing allows avoiding disturbance during the functioning of this approach. This paper has focused on vehicular traffic and on assessing the requirements of the user on the road. However, future investigation is suggested in order to find the suitable way to combine the appropiate computational methods to implement each one of the proposed components of UUTC.

\section{References}

[1] Weiser, M. (1991) The Computer for the 21st Century. Scientific American, 265, 94-104. http://dx.doi.org/10.1038/scientificamerican0991-94

[2] Satyanarayanan, M. (2001) Pervasive Computing: Vision and Challenges. IEEE Personal Communications, 8, 10-17. http://dx.doi.org/10.1109/98.943998

[3] Vianna, H.D. and Barbosa, J.L.V. (2014) A Model for Ubiquitous Care of Noncommunicable Diseases. IEEE Journal of Biomedical and Health Informatics, 18, 1597-1606. http://dx.doi.org/10.1109/JBHI.2013.2292860

[4] Franco, L.K., Rosa, J.H., Barbosa, J.L.V., Costa, C.A. and Cyamin, A. (2011) MUCS: A Model for Ubiquitous Commerce Support. Electronic Commerce Research and Applications, 10, 237-246. http://dx.doi.org/10.1016/j.elerap.2010.08.006

[5] Rosa, J.H., Barbosa, J.L.V., Kich, M.R. and Brito, L.K. (2015) A Multi-Temporal Context-Aware System for Competences Management. International Journal of Artificial Intelligence in Education, 25, 455-492. http://dx.doi.org/10.1007/s40593-015-0047-y

[6] Barbosa, J.L.V., Hahn, R., Barbosa, D.N.F. and Saccol, A.I.C.Z. (2011) A Ubiquitous Learning Model Focused on Learner Integration. International Journal of Learning Technology, 6, 62-83.

${ }^{2}$ https://www.arduino.cc.

${ }^{3}$ https://www.raspberrypi.org. 
http://dx.doi.org/10.1504/IJLT.2011.040150

[7] Oliveira, R.R., Noguez, F.C., Costa, C.A., Barbosa, J.L.V. and Prado, M.P. (2013) SWTRACK: An Intelligent Model for Cargo Tracking Based on Off-the-Shelf Mobile Devices. Expert Systems with Applications, 40, 2023-2031. http://dx.doi.org/10.1016/j.eswa.2012.10.021

[8] Oliveira, R.R., Cardoso, I.G., Barbosa, J.L.V., Costa, C.A. and Prado, M.P. (2015) An Intelligent Model for Logistics Management Based on Geofencing Algorithms and RFID Technology. Expert Systems with Applications, 42, 60826097. http://dx.doi.org/10.1016/j.eswa.2015.04.001

[9] Ibarra, S., Castán, J.A. and Laria, J. (2015) Optimizing Urban Traffic Control Using a Rational Agent. Journal of Zhejiang University SCIENCE C, 15, 1123-1137. http://link.springer.com/article/10.1631\%2Fjzus.C1400037

[10] Sharma, M. and Siddiqui, A. (2010) RFID Based Mobile: Next Generation Applications. The 2nd IEEE International Conference on Information Management and Engineering (ICIME), Chengdu, 16-18 April 2010, 523-526. http://dx.doi.org/10.1109/icime.2010.5477641

[11] Segatto, W., Herzer, E., Mazzotti, C.L., Bittencourt, J.R. and Barbosa, J.L.V. (2008) moBIO Threat: A Mobile Game Based on the Integration of Wireless Technologies. Computers in Entertainment, 6, Article No. 39. http://dx.doi.org/10.1145/1394021.1394032

[12] Victória, J.L. (2015) Ubiquitous Computing. Applications and Research Opportunities. IEEE International Conference on Computational Intelligence and Computing Research (ICCIC). Madurai, December 2015, 1-8.

[13] Husna, N. Faidzul, M. Rosmadi, M. and Modh, A (2015) Advanced Ubiquitous Computing to Support Samrt City, Smart Village Applications. 720-725.

[14] Luo, Z., Zhang, T. and Wang, C. (2011) RFID Enable Vehicular Wireless Query for Travel Information in Intelligent Transportation Systems. 2011 IEEE International Conference on RFID-Technologies and Applications, Sitges, Spain, 15-16 September 2011, 415-420.

[15] Nam, K., Choi, E., Park, J. and Park, H. (2011) Information Lifecycle Management in City-Wide Ubiquitous Computing Environment. 2011 IEEE 13th International Conference on High Performance Computing and Communications (HPCC), Banff, AB, 2-4 September 2011, 910-915. http://dx.doi.org/10.1109/hpcc.2011.131

[16] Gupta, A., Sharma, V., Kumar, N., Jain, S., Alhammad, A. and Kamal, A. (2014) Integrating Pervasive Computing, Infostations and Swarm Intelligence to Design Intelligent Context-Aware Parking Space Location Mechanism. 2014 International Conference on Advances in Computing, Communications and Informatics, New Delhi, 24-27 September 2014, 1381-1387. http://dx.doi.org/10.1109/icacci.2014.6968231

[17] Wang, C., David, B. and Chalon, R. (2014) Dynamic Road Lane Management: A Smart City Application. 2014 International Conference on Advanced Logistics and Transport (ICALT), Hammamet, 1-3 May 2014, 72-77. http://dx.doi.org/10.1109/icadlt.2014.6864085

[18] He, W., Tan, E.L., Lee, E.W. and Li, T.Y. (2009) A Solution for Integrated Track and Trace in Supply Chain Based on RFID and GPS. Proocedings of the IEEE Conference on Emerging Technologies and Factory Automation, Mallorca, Spain, September 2009, 1-6.

[19] Reclus, F. and Drouard, K. (2009) Geofencing for Fleet and Freight Management. Proceedings of the 9th International Conference on Intelligent Transport Systems Telecommunication, Lille, France, October 2009, 353-356.

\section{Submit or recommend next manuscript to SCIRP and we will provide best service for you:}

Accepting pre-submission inquiries through Email, Facebook, LinkedIn, Twitter, etc.

A wide selection of journals (inclusive of 9 subjects, more than 200 journals)

Providing 24-hour high-quality service

User-friendly online submission system

Fair and swift peer-review system

Efficient typesetting and proofreading procedure

Display of the result of downloads and visits, as well as the number of cited articles

Maximum dissemination of your research work

Submit your manuscript at: http://papersubmission.scirp.org/ 